\title{
Positive solutions for boundary value problem of fractional differential equation with $p$-Laplacian operator
}

\author{
Guoging Chai
}

Correspondence: mathchgq@gmail. com

College of Mathematics and Statistics, Hubei Normal University, Hubei 435002, P.R. China

\section{Abstract}

In this article, the author investigates the existence and multiplicity of positive solutions for boundary value problem of fractional differential equation with $p$ Laplacian operator

$$
\begin{cases}D_{0+}^{\beta}\left(\varphi_{p}\left(D_{0+}^{\alpha} u\right)\right)(t)+f(t, u(t))=0,0<t<1, \\ u(0)=0, u(1)+\sigma D_{0+}^{\gamma} u(1)=0, \quad D_{0+}^{\alpha} u(0)=0,\end{cases}
$$

where $D_{0+}^{\beta}, D_{0+}^{\alpha}$ and $D_{0+}^{\gamma}$ are the standard Riemann-Liouville derivatives with $1<\alpha \leq$ $2,0<\beta \leq 1,0<\gamma \leq 1,0 \leq \alpha-\gamma-1$, the constant $\sigma$ is a positive number and $p$ Laplacian operator is defined as $\phi_{p}(s)=|s|^{p-2} s, p>1$. By means of the fixed point theorem on cones, some existence and multiplicity results of positive solutions are obtained.

2010 Mathematical Subject Classification: 34A08; 34B18.

Keywords: fractional differential equations, fixed point index, p-Laplacian operator, positive solution, multiplicity of solutions

\section{Introduction}

Differential equations of fractional order have been recently proved to be valuable tools in the modeling of many phenomena in various fields of science and engineering. Indeed, we can find numerous applications in viscoelasticity, electrochemistry, control, porous media, electromagnetism, etc. (see [1-5]). There has been a significant development in the study of fractional differential equations in recent years, see the monographs of Kilbas et al. [6], Lakshmikantham et al. [7], Podlubny [4], Samko et al. [8], and the survey by Agarwal et al. [9].

For some recent contributions on fractional differential equations, see for example, [10-28] and the references therein. Especially, in [15], by means of Guo-Krasnosel'skiir's fixed point theorem, Zhao et al. investigated the existence of positive solutions for the nonlinear fractional boundary value problem (BVP for short)

$$
\left\{\begin{array}{l}
D_{0_{+}}^{\alpha} u(t)=\lambda f(u(t)), t \in(0,1), \\
u(0)+u^{\prime}(0)=0, \quad u(1)+u^{\prime}(1)=0,
\end{array}\right.
$$

where $1<\alpha \leq 2, f:[0,+\infty) \rightarrow(0,+\infty)$. 
In [16], relying on the Krasnosel'skiîs fixed point theorem as well as on the LeggettWilliams fixed point theorem, Kaufmann and Mboumi discussed the existence of positive solutions for the following fractional BVP

$$
\left\{\begin{array}{l}
D_{0+}^{\alpha} u(t)+a(t) f(u(t))=0, \quad 0<t<1, \quad 1<\alpha \leq 2, \\
u(0)=0, \quad u^{\prime}(1)=0 .
\end{array}\right.
$$

In [17], by applying Altman's fixed point theorem and Leray-Schauder' fixed point theorem, Wang obtained the existence and uniqueness of solutions for the following BVP of nonlinear impulsive differential equations of fractional order $q$

$$
\left\{\begin{array}{l}
{ }^{c} D^{q} u(t)=f(t, u(t)), \quad 1<q \leq 2, \quad t \in J^{\prime}, \\
\Delta u\left(t_{k}\right)=Q_{k}\left(u\left(t_{k}\right)\right), \quad \Delta u^{\prime}\left(t_{k}\right)=I_{k}\left(u\left(t_{k}\right)\right), \quad k=1,2, \ldots p, \\
\operatorname{au}(0)-b u^{\prime}(0)=x_{0}, c u(1)+d u^{\prime}(1)=x_{1} .
\end{array}\right.
$$

In [18], relying on the contraction mapping principle and the Krasnosel'skiî's fixed point theorem, Zhou and Chu discussed the existence of solutions for a nonlinear multi-point BVP of integro-differential equations of fractional order $q \in(1,2]$

$$
\left\{\begin{array}{l}
{ }^{c} D_{0_{+}}^{q} u(t)=f(t, u(t),(K u)(t),(H u)(t)), \quad 1<t<1, \\
a_{1} u(0)-b_{1} u^{\prime}(0)=d_{1} u\left(\xi_{1}\right), \quad a_{2} u(1)+b_{2} u^{\prime}(1)=u\left(\xi_{2}\right) .
\end{array}\right.
$$

On the other hand, integer-order $p$-Laplacian boundary value problems have been widely studied owing to its importance in theory and application of mathematics and physics, see for example, [29-33] and the references therein. Especially, in [29], by using the fixed point index method, Yang and Yan investigated the existence of positive solution for the third-order Sturm-Liouville boundary value problems with $p$ Laplacian operator

$$
\left\{\begin{array}{l}
\left(\phi_{p}\left(u^{\prime \prime}(t)\right)^{\prime}+f(t, u(t))=0, \quad t \in(0,1),\right. \\
a u(0)-b u^{\prime}(0)=0 \quad c u(1)+u^{\prime}(1)=0, \quad u^{\prime \prime}(0)=0,
\end{array}\right.
$$

where $\phi_{p}(s)=|s|^{p-2} s$.

However, there are few articles dealing with the existence of solutions to boundary value problems for fractional differential equation with $p$-Laplacian operator. In [24], the authors investigated the nonlinear nonlocal problem

$$
\left\{\begin{array}{l}
D_{0+}^{\beta}\left(\varphi_{p}\left(D_{0+}^{\alpha} u\right)\right)(t)+f(t, u(t))=0, \quad 0<t<1, \\
u(0)=0, u(1)=\mathrm{au}(\xi), \quad D_{0+}^{\alpha} u(0)=0,
\end{array}\right.
$$

where $0<\beta \leq 1,1<\alpha \leq 2,0 \leq a \leq 1,0<\xi<1$. By using Krasnosel'skiî's fixed point theorem and Leggett-Williams theorem, some sufficient conditions for the existence of positive solutions to the above BVP are obtained.

In [25], by using upper and lower solutions method, under suitable monotone conditions, the authors investigated the existence of positive solutions to the following nonlocal problem

$$
\left\{\begin{array}{l}
D_{0+}^{\beta}\left(\varphi_{p}\left(D_{0+}^{\alpha} u\right)\right)(t)+f(t, u(t)), \quad 0<t<1 \\
u(0)=0, u(1)=\mathrm{au}(\xi), \quad D_{0+}^{\alpha} u(0)=0, \quad D_{0+}^{\alpha} u(1)=b D_{0+}^{\alpha} u(\eta)
\end{array}\right.
$$

where $1<\alpha, \beta \leq 2,0 \leq a, b \leq 1,0<\xi, \eta<1$.

No contribution exists, as far as we know, concerning the existence of solutions for the fractional differential equation with $p$-Laplacian operator 


$$
\left\{\begin{array}{l}
D_{0+}^{\beta}\left(\varphi_{p}\left(D_{0+}^{\alpha} u\right)\right)(t)+f(t, u(t))=0,0<t<1, \\
u(0)=0, \quad u(1)+\sigma D_{0+}^{\gamma} u(1)=0, D_{0+}^{\alpha} u(0)=0,
\end{array}\right.
$$

where $D_{0^{+}}^{\beta}, D_{0+}^{\alpha}$ and $D_{0+}^{\gamma}$ are the standard Riemann-Liouville derivative with $1<\alpha \leq 2$, $0<\beta \leq 1,0<\gamma \leq 1,0 \leq \alpha-\gamma-1$, the constant $\sigma$ is a positive number, the $p$-Laplacian operator is defined as $\phi_{p}(s)=|s|^{p-2} s, p>1$, and function $f$ is assumed to satisfy certain conditions, which will be specified later. To obtain the existence and multiplicity of positive solutions to BVP (1.5), the fixed point theorem on cones will be applied.

It is worth emphasizing that our work presented in this article has the following features which are different from those in $[24,25]$. Firstly, BVP (1.5) is an important two point BVP. When $\gamma=1$, the boundary value conditions in (1.5) reduce to $u(0)=0, u$ $(1)+\sigma u^{\prime}(1)=0$, which are the well-known Sturm-Liouville boundary value conditions $u(0)+b u^{\prime}(0)=0, u(1)+\sigma u^{\prime}(1)=0$ (such as BVP (1.1)) with $b=0$. It is a well-known fact that the boundary value problems with Sturm-Liouville boundary value conditions for integral order differential equations have important physical and applied background and have been studied in many literatures, while BVPs (1.3) and (1.4) are the nonlocal boundary value problems, which are not able to substitute BVP (1.5). Secondly, when $\alpha=2, \beta=1, \gamma=1$, then BVP (1.5) reduces to BVP (1.2) with $b=0$. So, BVP (1.5) is an important generalization of BVP (1.2) from integral order to fractional order. Thirdly, in BVPs (1.3) or (1.4), the boundary value conditions $u(1)=\mathrm{au}(\xi)$, $D_{0+}^{\alpha} u(1)=b D_{0+}^{\alpha} u(\eta)$ show the relations between the derivatives of same order $D_{0+}^{\mu} u(1)$ and $D_{0+}^{\mu} u(\zeta)(\mu=0, \alpha)$. By contrast with that, the condition $u(1)+\sigma D_{0+}^{\gamma} u(1)=0$ in BVP (1.5) shows that relation between the derivatives of different order $u(1)$ and $D_{0+}^{\gamma} u(1)$ is regarded as the derivative value of zero order of $u$ at $t=1$ ), which brings about more difficulties in deducing the property of green's function than the former. Finally, order $\alpha+\beta$ satisfies that $2<\alpha+\beta \leq 4$ in BVP (1.4), while order $\alpha+\beta$ satisfies that $1<\alpha+\beta \leq 3$ in BVP (1.5). In the case for $\alpha, \beta$ taking integral numbers, the BVPs (1.5) and (1.4) are the third-order BVP and the fourth-order BVP, respectively. So, BVP (1.5) differs essentially from BVP (1.4). In addition, the conditions imposed in present paper are easily verified.

The organization of this article is as follows. In Section 2, we present some necessary definitions and preliminary results that will be used to prove our main results. In Section 3, we put forward and prove our main results. Finally, we will give two examples to demonstrate our main results.

\section{Preliminaries}

In this section, we introduce some preliminary facts which are used throughout this article.

Let $\mathbb{N}$ be the set of positive integers, $\mathbb{R}$ be the set of real numbers and $\mathbb{R}_{+}$be the set of nonnegative real numbers. Let $I=[0,1]$. Denote by $C(I, \mathbb{R})$ the Banach space of all continuous functions from $I$ into $\mathbb{R}$ with the norm

$$
\|u\|=\max \{|u(t)|: t \in I\} .
$$

Define the cone $P$ in $C(I, \mathbb{R})$ as $P=\{u \in C(I, \mathbb{R}): u(t) \geq 0, t \in I\}$. Let $q>1$ satisfy the relation $\frac{1}{q}+\frac{1}{p}=1$, where $p$ is given by (1.5). 
Definition 2.1. [6] The Riemann-Liouville fractional integral of order $\alpha>0$ of a function $y:(a, b] \rightarrow \mathbb{R}$ is given by

$$
I_{a+}^{\alpha} y(t)=\frac{1}{\Gamma(\alpha)} \int_{a}^{t}(t-s)^{\alpha-1} y(s) d s, \quad t \in(a, b] .
$$

Definition 2.2. [6] The Riemann-Liouville fractional derivative of order $\alpha>0$ of function $y:(a, b] \rightarrow \mathbb{R}$ is given by

$$
D_{a+}^{\alpha} \gamma(t)=\frac{1}{\Gamma(n-\alpha)}\left(\frac{d}{d t}\right)^{n} \int_{a}^{t} \frac{\gamma(s)}{(t-s)^{\alpha-n+1}} d s, \quad t \in(a, b],
$$

where $n=[\alpha]+1$ and $[\alpha]$ denotes the integer part of $\alpha$.

Lemma 2.1. [34] Let $\alpha>0$. If $u \in C(0,1) \cap L(0,1)$ possesses a fractional derivative of order $\alpha$ that belongs to $C(0,1) \cap L(0,1)$, then

$$
I_{0+}^{\alpha} D_{0+}^{\alpha} u(t)=u(t)+c_{1} t^{\alpha-1}+c_{2} t^{\alpha-2}+\cdots+c_{n} t^{\alpha-n},
$$

for some $c_{i} \in \mathbb{R}, i=1,2, \ldots, n$, where $n=[\alpha]+1$.

A function $u \in C(I, \mathbb{R})$ is called a nonnegative solution of BVP (1.5), if $u \geq 0$ on [0, $1]$ and satisfies (1.5). Moreover, if $u(t)>0, t \in(0,1)$, then $u$ is said to be a positive solution of BVP (1.5).

For forthcoming analysis, we first consider the following fractional differential equation

$$
\left\{\begin{array}{l}
D_{0_{+}}^{\alpha} u(t)+\phi(t)=0, \quad 0<t<1, \\
u(0)=0, \quad u(1)+\sigma D_{0+}^{\gamma} u(1)=0,
\end{array}\right.
$$

where $\alpha, \gamma, \sigma$ are given by (1.5) and $\varphi \in C(I, \mathbb{R})$.

By Lemma 2.1, we have

$$
u(t)=c_{1} t^{\alpha-1}+c_{2} t^{\alpha-2}-I_{0+}^{\alpha} \phi(t), \quad t \in[0,1] .
$$

From the boundary condition $u(0)=0$, we have $c_{2}=0$, and so

$$
u(t)=c_{1} t^{\alpha-1}-I_{0+}^{\alpha} \phi(t), \quad t \in[0,1] .
$$

Thus,

$$
D_{0+}^{\gamma} u(t)=c_{1} \frac{\Gamma(\alpha)}{\Gamma(\alpha-\gamma)} t^{\alpha-\gamma-1}-I_{0+}^{\alpha-\gamma} \phi(t)
$$

and

$$
u(1)=c_{1}-I_{0+}^{\alpha} \phi(1), \quad D_{0+}^{\gamma} u(1)=c_{1} \frac{\Gamma(\alpha)}{\Gamma(\alpha-\gamma)}-I_{0+}^{\alpha-\gamma} \phi(1) .
$$

From the boundary condition $u(1)+\sigma D_{0+}^{\gamma} u(1)=0$, it follows that

$$
\left(1+\sigma \frac{\Gamma(\alpha)}{\Gamma(\alpha-\gamma)}\right) c_{1}-\left(I_{0+}^{\alpha} \phi(1)+\sigma I_{0+}^{\alpha-\gamma} \phi(1)\right)=0 .
$$


Let $\delta=\left[1+\sigma \frac{\Gamma(\alpha)}{\Gamma(\alpha-\gamma)}\right]^{-1}$. Then

$$
c_{1}=\delta\left[I_{0+}^{\alpha} \phi(1)+\sigma I_{0+}^{\alpha-\gamma} \phi(1)\right] .
$$

Substituting (2.3) into (2.2), we have

$$
\begin{aligned}
u(t)= & \delta\left[I_{0+}^{\alpha} \phi(1)+\sigma I_{0+}^{\alpha-\gamma} \phi(1)\right] t^{\alpha-1}-I_{0+}^{\alpha} \phi(t) \\
= & \delta t^{\alpha-1}\left[\frac{1}{\Gamma(\alpha)} \int_{0}^{1}(1-s)^{\alpha-1} \phi(s) d s+\frac{1}{\Gamma(\alpha-\gamma)} \sigma \int_{0}^{1}(1-s)^{\alpha-\gamma-1} \phi(s) d s\right] \\
& -\frac{1}{\Gamma(\alpha)} \int_{0}^{t}(t-s)^{\alpha-1} \phi(s) d s \\
= & \frac{1}{\Gamma(\alpha)}\left\{\delta t^{\alpha-1} \int_{0}^{1}\left[(1-s)^{\alpha-1}+\frac{\sigma \Gamma(\alpha)}{\Gamma(\alpha-\gamma)}(1-s)^{\alpha-\gamma-1}\right] \phi(s) d s-\int_{0}^{t}(t-s)^{\alpha-1} \phi(s) d s\right\} \\
= & \frac{1}{\Gamma(\alpha)}\left\{\int_{0}^{t}\left[\delta t^{\alpha-1}\left((1-s)^{\alpha-1}+\frac{\sigma \Gamma(\alpha)}{\Gamma(\alpha-\gamma)}(1-s)^{\alpha-\gamma-1}\right)-(t-s)^{\alpha-1}\right] \phi(s) d s\right. \\
& \left.+\delta t^{\alpha-1} \int_{t}^{1}\left[(1-s)^{\alpha-1}+\frac{\sigma \Gamma(\alpha)}{\Gamma(\alpha-\gamma)}(1-s)^{\alpha-\gamma-1}\right] \phi(s) d s\right\} \\
= & \int_{0}^{1} G(t, s) \phi(s) d s,
\end{aligned}
$$

where

$$
G(t, s)=\frac{1}{\Gamma(\alpha)} \cdot\left\{\begin{array}{l}
g_{1}(t, s), 0 \leq s \leq t \leq 1 \\
g_{2}(t, s), 0 \leq t \leq s \leq 1
\end{array}\right.
$$

and

$$
\begin{aligned}
& g_{1}(t, s)=\delta t^{\alpha-1}\left[(1-s)^{\alpha-1}+\frac{\sigma \Gamma(\alpha)}{\Gamma(\alpha-\gamma)}(1-s)^{\alpha-\gamma-1}\right]-(t-s)^{\alpha-1}, \quad 0 \leq s \leq t, \\
& g_{2}(t, s)=\delta t^{\alpha-1}\left[(1-s)^{\alpha-1}+\frac{\sigma \Gamma(\alpha)}{\Gamma(\alpha-\gamma)}(1-s)^{\alpha-\gamma-1}\right], \quad t \leq s \leq 1 .
\end{aligned}
$$

So, we obtain the following lemma.

Lemma 2.2. The solution of Equation (2.1) is given by

$$
u(t)=\int_{0}^{1} G(t, s) \phi(s) d s, \quad t \in[0,1] .
$$

Also, we have the following lemma.

Lemma 2.3. The Green's function $G(t, s)$ has the following properties

(i) $G(t, s)$ is continuous on $[0,1] \times[0,1]$,

(ii) $G(t, s)>0, s, t \in(0,1)$.

Proof. (i) Owing to the fact $1<\alpha \leq 2,0<\gamma \leq 1,0 \leq \alpha-\gamma-1$, from the expression of $G$, it is easy to see that conclusion (i) of Lemma 2.3 is true. 
(ii) There are two cases to consider.

(1) If $0<s \leq t<1$, then

$$
\begin{aligned}
\Gamma(\alpha) g_{1}(t, s) & =t^{\alpha-1}\left\{\delta\left[(1-s)^{\alpha-1}+\frac{\sigma \Gamma(\alpha)}{\Gamma(\alpha-\gamma)}(1-s)^{\alpha-\gamma-1}\right]-\left(1-\frac{s}{t}\right)^{\alpha-1}\right\} \\
& >t^{\alpha-1}\left\{\delta\left[(1-s)^{\alpha-1}+\frac{\sigma \Gamma(\alpha)}{\Gamma(\alpha-\gamma)}(1-s)^{\alpha-\gamma-1}\right]-(1-s)^{\alpha-1}\right\} \\
& =t^{\alpha-1}(1-s)^{\alpha-1}\left\{\delta\left[1+\frac{\sigma \Gamma(\alpha)}{\Gamma(\alpha-\gamma)}(1-s)^{-\gamma}\right]-1\right\} \\
& \geq t^{\alpha-1}(1-s)^{\alpha-1}\left\{\delta\left[1+\frac{\sigma \Gamma(\alpha)}{\Gamma(\alpha-\gamma)}\right]-1\right\}=0 .
\end{aligned}
$$

(2) If $0<t \leq s<1$, then conclusion (ii) of Lemma 2.3 is obviously true from the expression of $G$.

We need to introduce some notations for the forthcoming discussion.

Let $\eta_{0}=\left(\frac{\gamma \delta \sigma \Gamma(\alpha)}{\Gamma(\alpha-\gamma)}\right)^{\frac{1}{\alpha-1}}$. Denote $\eta(s)=\frac{\gamma \delta \sigma \Gamma(\alpha)}{\Gamma(\alpha-\gamma)} s^{2-\alpha}, s \in[0,1]$. Set $g(s)=G(s$, $s), s \in[0,1]$. From $0<\gamma \leq 1, \sigma>0,1<\alpha \leq 2$ and $\delta=\left[1+\frac{\sigma \Gamma(\alpha)}{\Gamma(\alpha-\gamma)}\right]^{-1}$, we know that $\eta_{0} \in(0,1)$.

The following lemma is fundamental in this article.

Lemma 2.4. The Green's function $G$ has the properties

(i) $G(t, s) \leq G(s, s), s, t \in[0,1]$.

(ii) $G(t, s) \geq \eta(s) G(s, s), t \in\left[\eta_{0}, 1\right], s \in[0,1]$.

Proof. (i) There are two cases to consider.

Case 1. $0 \leq s \leq t \leq 1$. In this case, since the following relation

$$
\begin{aligned}
\frac{\partial g_{1}(t, s)}{\partial t} & =(\alpha-1)\left\{\delta t^{\alpha-2}\left[(1-s)^{\alpha-1}+\frac{\sigma \Gamma(\alpha)}{\Gamma(\alpha-\gamma)}(1-s)^{\alpha-\gamma-1}\right]-(t-s)^{\alpha-2}\right\} \\
& \leq(\alpha-1)\left\{\delta t^{\alpha-2}\left[1+\frac{\sigma \Gamma(\alpha)}{\Gamma(\alpha-\gamma)}\right]-(t-s)^{\alpha-2}\right\} \\
& =(\alpha-1)\left\{t^{\alpha-2}-(t-s)^{\alpha-2}\right\}<0 .
\end{aligned}
$$

holds for $0<s<t \leq 1$, we have

$$
G(t, s) \leq G(s, s), \quad 0 \leq s \leq t \leq 1 .
$$

Case 2. $0 \leq t \leq s \leq 1$. In this case, from the expression of $g_{2}(t, s)$, it is easy to see that

$$
G(t, s) \leq G(s, s), \quad 0 \leq t \leq s \leq 1 .
$$

(ii) We will consider the following two cases.

Case 1. When $0<s \leq \eta_{0}, \eta_{0} \leq t \leq 1$, then from the above argument in (i) of proof, we know that $g_{1}(t, s)$ is decreasing with respect to $t$ on $\left[\eta_{0}, 1\right]$. Thus

$$
\min _{t \in\left[\eta_{0}, 1\right]} G(t, s)=G(1, s)=g_{1}(1, s) / \Gamma(\alpha), \quad s \in(0, \eta 0]
$$


and so

$$
\min _{t \in\left[\eta_{0}, 1\right]} \frac{G(t, s)}{G(s, s)}=\frac{g_{1}(1, s)}{g(s)}, \quad \text { for } s \in(0, \eta 0] .
$$

Case 2. $\eta_{0}<s<1, \eta_{0} \leq t \leq 1$.

(a) If $s \leq t$, then by similar arguments to (2.5), we also have

$$
\min _{t \in[s, 1]} G(t, s)=G(1, s)=g_{1}(1, s) / \Gamma(\alpha) .
$$

(b) If $\eta_{0} \leq t \leq s$, then the following relation

$$
\min _{t \in\left[\eta_{0}, s\right]} G(t, s)=g_{2}\left(\eta_{0}, s\right) / \Gamma(\alpha)
$$

holds in view of the expression of $g_{2}(t, s)$.

To summarize,

$$
\min _{t \in\left[\eta_{0}, 1\right]} \frac{G(t, s)}{G(s, s)} \geq \min \left\{\frac{g_{1}(1, s)}{g(s)}, \frac{g_{2}\left(\eta_{0}, s\right)}{g(s)}\right\}, \quad \text { for all } s \in\left(\eta_{0}, 1\right)
$$

Now, we shall show that

$$
\min _{t \in\left[\eta_{0}, 1\right]} \frac{G(t, s)}{G(s, s)} \geq \frac{\gamma \delta \sigma \Gamma(\alpha)}{\Gamma(\alpha-\gamma)} s^{2-\alpha}, s \in(0,1) .
$$

In fact, for $s \in(0,1)$, we have

$$
\begin{aligned}
g_{1}(1, s)= & \delta\left[(1-s)^{\alpha-1}+\frac{\sigma \Gamma(\alpha)}{\Gamma(\alpha-\gamma)}(1-s)^{\alpha-\gamma-1}\right]-(1-s)^{\alpha-1} \\
= & \delta\left[(1-s)^{\alpha-1}+\frac{\sigma \Gamma(\alpha)}{\Gamma(\alpha-\gamma)}(1-s)^{\alpha-1}\right] \\
& +\delta \frac{\sigma \Gamma(\alpha)}{\Gamma(\alpha-\gamma)}\left[(1-s)^{\alpha-\gamma-1}-(1-s)^{\alpha-1}\right]-(1-s)^{\alpha-1} \\
= & \delta\left[1+\frac{\sigma \Gamma(\alpha)}{\Gamma(\alpha-\gamma)}\right](1-s)^{\alpha-1} \\
& \left.+\delta \frac{\sigma \Gamma(\alpha)}{\Gamma(\alpha-\gamma)}(1-s)^{\alpha-\gamma-1}\left[1-(1-s)^{\gamma}\right)\right]-(1-s)^{\alpha-1} \\
= & \delta \frac{\sigma \Gamma(\alpha)}{\Gamma(\alpha-\gamma)}(1-s)^{\alpha-\gamma-1}\left[1-(1-s)^{\gamma}\right] \\
> & \delta \frac{\sigma \Gamma(\alpha)}{\Gamma(\alpha-\gamma)}(1-s)^{\alpha-\gamma-1} \gamma s,
\end{aligned}
$$

and so

$$
\begin{aligned}
\frac{g_{1}(1, s)}{g(s)}> & \frac{\gamma \delta \frac{\sigma \Gamma(\alpha)}{\Gamma(\alpha-\gamma)}(1-s)^{\alpha-\gamma-1} s}{\delta s^{\alpha-1}\left[(1-s)^{\alpha-1}+\frac{\sigma \Gamma(\alpha)}{\Gamma(\alpha-\gamma)}(1-s)^{\alpha-\gamma-1}\right]} \\
= & \frac{\gamma \frac{\sigma \Gamma(\alpha)}{\Gamma(\alpha-\gamma)} s^{2-\alpha}}{(1-s)^{\gamma}+\frac{\sigma \Gamma(\alpha)}{\Gamma(\alpha-\gamma)}} \\
> & \frac{\gamma \frac{\sigma \Gamma(\alpha)}{\Gamma(\alpha-\gamma)} s^{2-\alpha}}{1+\frac{\sigma \Gamma(\alpha)}{\Gamma(\alpha-\gamma)}} \\
= & \frac{\gamma \delta \sigma \Gamma(\alpha)}{\Gamma(\alpha-\gamma)} s^{2-\alpha}, \quad s \in(0,1) .
\end{aligned}
$$


On the other hand, for $s \in\left(\eta_{0}, 1\right)$, we have

$$
\frac{g_{2}\left(\eta_{0}, s\right)}{g(s)}=\eta_{0}^{\alpha-1} s^{1-\alpha}
$$

Since $\eta_{0}^{\alpha-1}=\frac{\gamma \delta \sigma \Gamma(\alpha)}{\Gamma(\alpha-\gamma)}$, the equality

$$
\eta_{0}^{\alpha-1} s^{1-\alpha}=\frac{\gamma \delta \sigma \Gamma(\alpha)}{\Gamma(\alpha-\gamma)} s^{2-\alpha}
$$

holds for $s=1$. Thus,

$$
\eta_{0}^{\alpha-1} s^{1-\alpha}>\frac{\gamma \delta \sigma \Gamma(\alpha)}{\Gamma(\alpha-\gamma)} s^{2-\alpha}, \quad s \in(0,1) .
$$

Since $1<\alpha \leq 2$, it follows from (2.9) that

$$
\frac{g_{2}\left(\eta_{0}, s\right)}{g(s)}>\frac{\gamma \delta \sigma \Gamma(\alpha)}{\Gamma(\alpha-\gamma)} s^{2-\alpha}, \quad s \in\left(\eta_{0}, 1\right) .
$$

Hence, from (2.8) and (2.11), we immediately have

$$
\min \left\{\frac{g_{1}(1, s)}{g(s)}, \frac{g_{2}\left(\eta_{0}, s\right)}{g(s)}\right\}>\frac{\gamma \delta \sigma \Gamma(\alpha)}{\Gamma(\alpha-\gamma)} s^{2-\alpha}, \quad s \in\left(\eta_{0}, 1\right) .
$$

Thus, from (2.6) and (2.12), it follows that

$$
\min _{t \in\left[\eta_{0}, 1\right]} \frac{G(t, s)}{G(s, s)}>\frac{\gamma \delta \sigma \Gamma(\alpha)}{\Gamma(\alpha-\gamma)} s^{2-\alpha}, \quad s \in\left(\eta_{0}, 1\right) .
$$

Also, by (2.8), the following inequality

$$
\frac{g_{1}(1, s)}{g(s)}>\frac{\gamma \delta \sigma \Gamma(\alpha)}{\Gamma(\alpha-\gamma)} s^{2-\alpha}, \quad s \in\left(0, \eta_{0}\right]
$$

holds, and therefore

$$
\min _{t \in\left[\eta_{0}, 1\right]} \frac{G(t, s)}{G(s, s)}>\frac{\gamma \delta \sigma \Gamma(\alpha)}{\Gamma(\alpha-\gamma)} s^{2-\alpha}, \quad s \in\left(0, \eta_{0}\right]
$$

from the proof in Case 1.

Summing up the above relations (2.13)-(2.14), we have

$$
\min _{t \in\left[\eta_{0}, 1\right]} \frac{G(t, s)}{G(s, s)}>\frac{\gamma \delta \sigma \Gamma(\alpha)}{\Gamma(\alpha-\gamma)} s^{2-\alpha}, \quad s \in(0,1),
$$

and so

$$
\min _{t \in\left[\eta_{0}, 1\right]} G(t, s) \geq \eta(s) G(s, s), \quad s \in[0,1] .
$$

The proof of Lemma 2.4 is complete.

To study BVP (1. 5), we first consider the associated linear BVP

$$
\left\{\begin{array}{l}
D_{0+}^{\beta}\left(\varphi_{p}\left(D_{0+}^{\alpha} u\right)\right)(t)+h(t)=0, \quad 0<t<1, \\
u(0)=0, \quad u(1)+\sigma D_{0+}^{\gamma} u(1)=0, \quad D_{0+}^{\alpha} u(0)=0,
\end{array}\right.
$$

where $h \in P$. 
Let $w=D_{0+}^{\alpha} u, v=\varphi_{p}(w)$. By Lemma 2.1, the solution of initial value problem

$$
\left\{\begin{array}{l}
D_{0+}^{\beta} u(t)+h(t)=0, t \in(0,1) \\
v(0)=0
\end{array}\right.
$$

is given by

$$
v(t)=C_{1} t^{\beta-1}-I_{0+}^{\beta} h(t), \quad t \in(0,1] .
$$

From the relations $v(0)=0,0<\beta \leq 1$, it follows that $C_{1}=0$, and so

$$
v(t)=-I_{0+}^{\beta} h(t), \quad t \in[0,1] .
$$

Noting that $D_{0+}^{\alpha} u=w, w=\varphi_{p}^{-1}(v)$, from (2.16), we know that the solution of (2.15) satisfies

$$
\left\{\begin{array}{l}
\left.D_{0+}^{\alpha} u(t)=\varphi_{p}^{-1}\left(-I_{0}^{\beta} h\right)(t)\right), \quad t \in(0,1) \\
u(0)=0, \quad u(1)+\sigma D_{0+}^{\gamma} u(1)=0 .
\end{array}\right.
$$

By Lemma 2.2, the solution of Equation (2.17) can be written as

$$
u(t)=-\int_{0}^{1} G(t, s) \varphi_{p}^{-1}\left(-I_{0}^{\beta} h\right)(s) d s, \quad t \in I .
$$

Since $h(s) \geq 0, s \in[0,1]$, we have $\varphi_{p}^{-1}\left(-I_{0+}^{\beta} h(s)\right)=-\left(\left(I_{0+}^{\beta} h\right)(s)\right)^{q-1}, s \in[0,1]$, and so

$$
\begin{aligned}
u(t) & =\int_{0}^{1} G(t, s)\left(I_{0+}^{\beta} h(s)\right)^{q-1} d s \\
& =\frac{1}{(\Gamma(\beta))^{q-1}} \int_{0}^{1} G(t, s) d s\left(\int_{0}^{s} h(\tau)(s-\tau)^{\beta-1} d \tau\right)^{q-1}
\end{aligned}
$$

from (2.18). Thus, by Lemma 2.3, we have obtained the following lemma.

Lemma 2.5. Let $h \in P$. Then the solution of Equation (2.15) in $P$ is given by

$$
u(t)=\frac{1}{(\Gamma(\beta))^{q-1}} \int_{0}^{1} G(t, s) d s\left(\int_{0}^{s} h(\tau)(s-\tau)^{\beta-1} d \tau\right)^{q-1} .
$$

We also need the following lemmas to obtain our results.

Lemma 2.6. If $a, b \geq 0, \gamma>0$, then

$$
(a+b)^{\gamma} \leq \max \left\{2^{\gamma-1}, 1\right\}\left(a^{\gamma}+b^{\gamma}\right) .
$$

Proof. Obviously, without loss of generality, we can assume that $0<a<b, \gamma \neq 1$.

Let $\varphi(t)=t^{\gamma}, t \in[0,+\infty)$.

(i) If $\gamma>1$, then $\varphi(t)$ is convex on $(0,+\infty)$, and so

$$
\phi\left(\frac{1}{2} a+\frac{1}{2} b\right) \leq \frac{1}{2} \phi(a)+\frac{1}{2} \phi(b),
$$




$$
\begin{aligned}
& \text { i.e., } \frac{1}{2^{\gamma}}(a+b)^{\gamma} \leq \frac{1}{2}\left(a^{\gamma}+b^{\gamma}\right) \text {. Thus } \\
& (a+b)^{\gamma} \leq 2^{\gamma-1}\left(a^{\gamma}+b^{\gamma}\right) .
\end{aligned}
$$

(ii) If $0<\gamma<1$, then $\varphi(t)$ is concave on $[0,+\infty)$, and so

$$
\begin{aligned}
\phi(a) & =\phi\left(\frac{b}{a+b} \cdot 0+\frac{a}{a+b} \cdot(a+b)\right) \\
& \geq \frac{b}{a+b} \phi(0)+\frac{a}{a+b} \cdot \phi(a+b) \\
& =\frac{a}{a+b} \phi(a+b), \\
\phi(b) & =\phi\left(\frac{b}{a+b} \cdot 0+\frac{a}{a+b} \cdot(a+b)\right) \\
& \geq \frac{a}{a+b} \phi(0)+\frac{b}{a+b} \phi(a+b) \\
& =\frac{b}{a+b} \phi(a+b) .
\end{aligned}
$$

Thus, $\varphi(a)+\varphi(b) \geq \varphi(a+b)$, namely,

$$
(a+b)^{\gamma} \leq a^{\gamma}+b^{\gamma}
$$

By (i), (ii) above, we know that the conclusion of Lemma 2.6 is true.

Lemma 2.7. Let $c>0, \gamma>0$. For any $x, y \in[0, c]$, we have that

(i) If $\gamma>1$, then $\left|x^{\gamma}-y^{\gamma}\right| \leq \gamma c^{\gamma-1}|x-y|$,

(ii) If $0<\gamma \leq 1$, then $\left|x^{\gamma}-y^{\gamma}\right| \leq|x-y|^{\gamma}$.

Proof. Obviously, without loss of generality, we can assume that $0<y<x$ since the variables $x$ and $y$ are symmetrical in the above inequality.

(i) If $\gamma>1$, then we set $\varphi(t)=t^{\gamma}, t \in[0, c]$. by virtue of mean value theorem, there exists a $\xi \in(0, c)$ such that

$$
\begin{aligned}
x^{\gamma}-y^{\gamma} & =\gamma \xi^{\gamma-1}(x-\gamma) \\
& \leq \gamma c^{\gamma-1}(x-\gamma),
\end{aligned}
$$

i.e.,

$$
\left|x^{\gamma}-y^{\gamma}\right| \leq \gamma c^{\gamma-1}|x-y| .
$$

(ii) If $0<\gamma<1$, then by Lemma 2.6, it is easy to see that

$$
\begin{aligned}
x^{\gamma}-y^{\gamma} & =(x-y-\gamma)^{\gamma}-y^{\gamma} \\
& \leq(x-y)^{\gamma}+y^{\gamma}-y^{\gamma}=(x-\gamma)^{\gamma},
\end{aligned}
$$

and so

$$
\left|x^{\gamma}-y^{\gamma}\right| \leq|x-y|^{\gamma}
$$

Now we introduce some notations, which will be used in the sequel. 
Let $D=\int_{0}^{1} G(s, s) s^{\beta(q-1)} d s, Q=\int_{0}^{1} \eta(s) G(s, s) s^{\beta(q-1)} d s$,

$$
l=\Gamma(\beta+1)\left(D \cdot \max \left\{2^{q-1}, 1\right\}\right)^{\frac{1}{1-q}}, \mu=\frac{\Gamma(\beta+1)}{Q^{\frac{1}{q-1}}} .
$$

By simple calculation, we know that

$$
\begin{aligned}
& D=\frac{\delta}{\Gamma(\alpha)} B(\alpha, \alpha+\beta(q-1))+\frac{\sigma \delta}{\Gamma(\alpha-\gamma)} B(\alpha-\gamma, \alpha+\beta(q-1)), \\
& Q=\frac{\gamma \delta^{2} \sigma}{\Gamma(\alpha-\gamma)}\left[B(\alpha, 2+\beta(q-1))+\frac{\sigma \Gamma(\alpha)}{\Gamma(\alpha-\gamma)} B(\alpha-\gamma, 2+\beta(q-1))\right] .
\end{aligned}
$$

In this article, the following hypotheses will be used.

$\left(H_{1}\right) f \in C\left(I \times \mathbb{R}_{+}, \mathbb{R}_{+}\right)$.

$\left(H_{2}\right) \varlimsup_{x \rightarrow+\infty} \max _{t \in I} \frac{f(t, x)}{x^{p-1}}<l, \lim _{x \rightarrow 0+} \min _{t \in I} \frac{f(t, x)}{x^{p-1}}>\mu$.

$\left(H_{3}\right)$ There exists a $r_{0}>0$ such that $f(t, x)$ is nonincreasing relative to $x$ on $\left[0, r_{0}\right]$ for any fixed $t \in I$.

By Lemma 2.5, it is easy to know that the following lemma is true.

Lemma 2.8. If $\left(H_{1}\right)$ holds, then BVP (1.5) has a nonnegative solution if and only if the integral equation

$$
u(t)=\frac{1}{(\Gamma(\beta))^{q-1}} \int_{0}^{1} G(t, s)\left(\int_{0}^{s} f(\tau, u(\tau))(s-\tau)^{\beta-1} d \tau\right)^{q-1} d s, \quad t \in I
$$

has a solution in $P$. Let $c$ be a positive number, $P$ be a cone and $P_{c}=\{y \in P:\|y\| \leq$ c). Let $\alpha$ be a nonnegative continuous concave function on $P$ and

$$
P(\alpha, a, b)=\{u \in P \mid a \leq \alpha(u),\|u\| \leq b\} .
$$

We will use the following lemma to obtain the multiplicity results of positive solutions.

Lemma 2.9. [35] Let $A: \overline{P_{c}} \rightarrow \overline{P_{c}}$ be completely continuous and $\alpha$ be a nonnegative continuous concave function on $P$ such that $\alpha(y) \leq\|y\|$ for all $y \in \overline{P_{c}}$. Suppose that there exist $a, b$ and $d$ with $0<a<b<d \leq c$ such that

(C1) $\{y \in P(\alpha, b, d)\} \mid \alpha(\gamma)>b\} \neq \emptyset$ and $\alpha(A y)>b$, for all $y \in P(\alpha, b, d)$;

(C2) $\|A y\|<a$, for $\|y\| \leq a$;

(C3) $\alpha(A y)>b$, for $y \in P(\alpha, b, c)$ with $\|A y\|>d$.

Then $A$ has at least three fixed points $y_{1}, y_{2}, y_{3}$ satisfying

$$
\left\|y_{1}\right\|<a, b<\alpha\left(y_{2}\right) \text {, and }\left\|y_{3}\right\|>a \text { with } \alpha\left(y_{3}\right)<b .
$$

\section{Main results}

In this section, our objective is to establish existence and multiplicity of positive solution to the BVP (1.5). To this end, we first define the operator on $P$ as 


$$
A u=\frac{1}{(\Gamma(\beta))^{q-1}} \int_{0}^{1} G(t, s)\left(\int_{0}^{s} f(\tau, u(\tau))(s-\tau)^{\beta-1} d \tau\right)^{q-1} d s, \quad u \in P .
$$

The properties of the operator $A$ are given in the following lemma.

Lemma 3.1. Let $\left(H_{1}\right)$ hold. Then $A: P \rightarrow P$ is completely continuous.

Proof. First, under assumption $\left(H_{1}\right)$, it is obvious that $A P \subset P$ from Lemma 2.3. Next, we shall show that operator $A$ is completely continuous on $P$. Let $E=\int_{0}^{1} G(s, s) d s$. The following proof will be divided into two steps.

Step 1. We shall show that the operator $A$ is compact on $P$.

Let $B$ be an arbitrary bounded set in $P$. Then exists an $M>0$ such that $\|u\| \leq M$ for

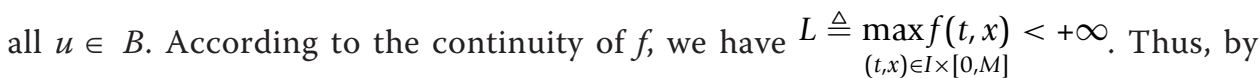
Lemmas 2.3 and 2.4, it follows that

$$
\begin{aligned}
0 \leq(\mathrm{A} u)(t) & \leq \frac{L^{q-1}}{(\Gamma(\beta))^{q-1}} \int_{0}^{1} G(s, s)\left(\int_{0}^{s}(s-\tau)^{\beta-1} d \tau\right)^{q-1} d s \\
& <\frac{L^{q-1}}{(\Gamma(\beta+1))^{q-1}} \int_{0}^{1} G(s, s) d s \\
& =\frac{L^{q-1}}{(\Gamma(\beta+1))^{q-1}} E, \quad t \in I .
\end{aligned}
$$

Thus,

$$
\|\mathrm{A} u\| \leq \frac{L^{q-1}}{(\Gamma(\beta+1))^{q-1}} E .
$$

That is, the set $A B$ is uniformly bounded.

On the other hand, the uniform continuity of $G(t, s)$ on $I \times I$ implies that for arbitrary $\varepsilon>0$, there exists a $\delta>0$ such that whenever $t_{1}, t_{2} \in I$ with $\left|t_{1}-t_{2}\right|<\delta$, the following inequality

$$
\left|G\left(t_{1}, s\right)-G\left(t_{2}, s\right)\right|<\varepsilon \frac{(\Gamma(\beta+1))^{q-1}}{L^{q-1}}(\beta(q-1)+1)
$$

holds for all $s \in I$. Therefore,

$$
\begin{aligned}
\left|\mathrm{A} u\left(t_{1}\right)-\mathrm{A} u\left(t_{2}\right)\right| & \leq \frac{1}{(\Gamma(\beta))^{q-1}} \int_{0}^{1}\left|G\left(t_{1}, s\right)-G\left(t_{2}, s\right)\right|\left(\int_{0}^{s} f(\tau, u(\tau))(s-\tau)^{\beta-1} d \tau\right)^{q-1} d s \\
& \leq \frac{L^{q-1}}{(\Gamma(\beta+1))^{q-1}} \int_{0}^{1} s^{(q-1) \beta} d s \frac{(\Gamma(\beta+1))^{q-1}}{L^{q-1}}(\beta(q-1)+1) \varepsilon=\varepsilon .
\end{aligned}
$$

Thus, $A B$ is equicontinuous. Consequently, the operator is compact on $P$ by ArzelàAscoli theorem.

Step 2. The operator $A$ is continuous.

Let $\left\{u_{n}\right\}$ be an arbitrary sequence in $P$ with $u_{n} \rightarrow u_{0} \in P$. Then exists an $L>0$ such that 


$$
0 \leq f\left(\tau, u_{n}(\tau)\right) \leq L, \quad \tau \in[0,1], \quad n \geq 0 .
$$

Thus,

$$
\int_{0}^{s} f\left(\tau, u_{n}(\tau)\right)(s-\tau)^{\beta-1} d \tau \leq L \int_{0}^{s}(s-\tau)^{\beta-1} d \tau \leq \frac{L}{\beta} \triangleq c, \quad s \in[0,1] .
$$

On the other hand, the uniform continuity of $f$ combined with the fact that $\left\|u_{n}-u_{0}\right\|$ $\rightarrow 0$ yields that there exists a $N \geq 1$ such that the following estimate

$$
\mid f\left(\tau, u_{n}(\tau)-f\left(t, u_{0}(\tau)\right) \mid<\varepsilon\right.
$$

holds for $n \geq N$.

(1) If $1<q \leq 2$, then from Lemma 2.7 (ii), we have

$$
\begin{aligned}
& \left|\left(\int_{0}^{s} f\left(\tau, u_{n}(\tau)\right)(s-\tau)^{\beta-1} d \tau\right)^{q-1}-\left(\int_{0}^{s} f\left(\tau, u_{0}(\tau)\right)(s-\tau)^{\beta-1} d \tau\right)^{q-1}\right| \\
& \leq\left(\int_{0}^{s}\left|f\left(\tau, u_{n}(\tau)\right)-f\left(\tau, u_{0}(\tau)\right)\right|(s-\tau)^{\beta-1} d \tau\right)^{q-1} \\
& <\varepsilon^{q-1} \frac{1}{\beta^{q-1}} s^{\beta(q-1)}, \quad s \in[0,1] .
\end{aligned}
$$

Hence, by Lemmas 2.3 and 2.4, from (3.1), we obtain

$$
\begin{aligned}
\left|\mathrm{A} u_{n}(t)-\mathrm{A} u_{0}(t)\right| & <\frac{\varepsilon^{q-1}}{(\Gamma(\beta+1))^{q-1}} \int_{0}^{1} G(s, s) d s \\
& =\frac{E}{(\Gamma(\beta+1))^{q-1}} \varepsilon^{q-1} .
\end{aligned}
$$

Thus,

$$
\left\|\mathrm{A} u_{n}-\mathrm{A} u_{0}\right\| \leq \frac{E}{(\Gamma(\beta+1))^{q-1}} \varepsilon^{q-1} .
$$

(2) If $q>2$, then from Lemma 2.7 (i), we have

$$
\begin{aligned}
& \left|\left(\int_{0}^{s} f\left(\tau, u_{n}(\tau)\right)(s-\tau)^{\beta-1} d \tau\right)^{q-1}-\left(\int_{0}^{s} f\left(\tau, u_{0}(\tau)\right)(s-\tau)^{\beta-1} d \tau\right)^{q-1}\right| \\
& \leq(q-1) c^{q-2} \int_{0}^{s}\left|f\left(\tau, u_{n}(\tau)\right)-f\left(\tau, u_{0}(\tau)\right)\right|(s-\tau)^{\beta-1} d \tau \\
& <\frac{q-1}{\beta} c^{q-2} s^{\beta} \varepsilon, \quad s \in[0,1] .
\end{aligned}
$$


Thus, we have

$$
\begin{aligned}
\left|\mathrm{A} u_{n}(t)-\mathrm{A} u_{0}(t)\right| & <\frac{(q-1) c^{q-2} \varepsilon}{\beta(\Gamma(\beta))^{q-1}} \int_{0}^{1} G(s, s) d s \\
& =\frac{(q-1) c^{q-2} E}{\beta(\Gamma(\beta))^{q-1}} \varepsilon
\end{aligned}
$$

and so

$$
\left\|\mathrm{A} u_{n}-\mathrm{A} u_{0}\right\| \leq \frac{(q-1) c^{q-2} E}{\beta(\Gamma(\beta))^{q-1}} \varepsilon .
$$

From (3.2)-(3.3), it follows that $\left\|A u_{n}-A u_{0}\right\| \rightarrow 0(n \rightarrow \infty)$.

Summing up the above analysis, we obtain that the operator $A$ is completely continuous on $P$.

We are now in a position to state and prove the first theorem in this article.

Theorem 3.1. Let $\left(H_{1}\right),\left(H_{2}\right)$, and $\left(H_{3}\right)$ hold. Then BVP (1.5) has at least one positive solution.

Proof. By Lemma 2.8, it is easy to know that BVP (1.5) has a nonnegative solution if and only if the operator $A$ has a fixed point in $P$. Also, we know that $A: P \rightarrow P$ is completely continuous by Lemma 3.1.

The following proof is divided into two steps.

Step 1. From $\left(H_{2}\right)$, we can choose a $\varepsilon_{0} \in(0, l)$ such that

$$
\varlimsup_{x \rightarrow+\infty} \max _{t \in I} \frac{f(t, x)}{x^{p-1}}<l-\varepsilon_{0} .
$$

Therefore, there exists a $R_{0}>0$ such that the inequality

$$
f(t, x)<\left(l-\varepsilon_{0}\right) x^{p-1}, \quad t \in I
$$

holds for $x \geq R_{0}$.

Let $M=\max _{(t, x) \in I \times\left[0, R_{0}\right]} f(t, x)$. It follows from (3.4) that

$$
f(t, x) \leq\left(l-\varepsilon_{0}\right) x^{p-1}+M, \quad \forall x \in \mathbb{R}_{+}, \quad t \in I .
$$

From the fact that $\left(l-\varepsilon_{0}\right)^{q-1}<l^{q-1}$, we can choose a $k>0$ such that $\left(l-\varepsilon_{0}\right)^{q-1}<l^{q-1}-k$. Set

$$
D_{1}=\frac{\max \left\{2^{q-2}, 1\right\} D}{(\Gamma(\beta+1))^{q-1}}, E=D_{1} k, G=D_{1} M^{q-1}
$$

where $D$ is as (2.20). Take $R>\frac{G}{E}$. Set $\Omega_{R}=\{u \in P:\|u\|<R\}$. We shall show that the relation

$$
\mathrm{A} u \neq \mu u, \quad \forall u \in \partial \Omega_{R} \quad \mu \geq 1
$$

holds.

In fact, if not, then there exists a $u_{0} \in \partial \Omega_{R}$ and a $\mu_{0} \geq 1$ with

$$
\mu_{0} u_{0}=\mathrm{A} u_{0} .
$$


By (3.5), we have

$$
\begin{aligned}
f\left(t, u_{0}(t)\right) & \leq\left(l-\varepsilon_{0}\right) u_{0}^{p-1}(t)+M \\
& \leq\left(l-\varepsilon_{0}\right)\left\|u_{0}\right\|^{p-1}+M \\
& =\left(l-\varepsilon_{0}\right) R^{p-1}+M, \quad t \in I .
\end{aligned}
$$

Therefore, in view of Lemmas 2.3, 2.4, from (3.1), it follows that

$$
\begin{aligned}
A u(t) & \leq \frac{\left(\left(l-\varepsilon_{0}\right) R^{p-1}+M\right)^{q-1}}{(\Gamma(\beta+1))^{q-1}} \int_{0}^{1} G(s, s) s^{\beta(q-1)} d s \\
& =\frac{D}{(\Gamma(\beta+1))^{q-1}}\left(\left(l-\varepsilon_{0}\right) R^{p-1}+M\right)^{q-1}, \quad t \in I .
\end{aligned}
$$

Also, keeping in mind that $(p-1)(q-1)=1$, by Lemma 2.6, we have

$$
\begin{aligned}
\left(\left(l-\varepsilon_{0}\right) R^{p-1}+M\right)^{q-1} & \leq \max \left\{2^{q-2}, 1\right\}\left(\left(l-\varepsilon_{0}\right)^{q-1} R+M^{q-1}\right) \\
& <\max \left\{2^{q-2}, 1\right\}\left(\left(l^{q-1}-k\right) R+M^{q-1}\right) .
\end{aligned}
$$

Hence, from (3.6), (3.8), and (3.9), it follows that

$$
u_{0} \leq \mu u_{0}=\mathrm{A} u_{0} \leq\left(D_{1} l^{q-1}-E\right) R+G, \quad t \in I .
$$

By definition of $l$, we have $D_{1} l^{q-1}=1$. From (3. 10), it follows that $R=\left\|u_{0}\right\| \leq(1-E) R$ $+G$, and so $R \leq \frac{G}{E}$, which contradicts the choice of $R$. Hence, the condition (3.7) holds. By virtue of the fixed point index theorem, we have

$$
i\left(A, \Omega_{R}, P\right)=1 .
$$

Step 2. By $\left(H_{2}\right)$, we can choose a $\varepsilon_{0}>0$ such that

$$
\varliminf_{x \rightarrow 0+} \min _{t \in I} \frac{f(t, x)}{x^{p-1}}>\mu+\varepsilon_{0}
$$

Hence, there exists a $r_{1} \in\left(0, r_{0}\right)$ such that

$$
f(t, x)>\left(\mu+\varepsilon_{0}\right) x^{p-1}, \quad t \in I, \quad x \in\left[0, r_{1}\right],
$$

where $r_{0}$ is given by $\left(H_{3}\right)$.

Take $0<r<\min \left\{R, r_{1}\right\}$, and set $\Omega_{r}=\{u \in P:\|u\|<r\}$. Now, we show that

(i) $\inf _{u \in \partial \Omega_{r}}\|\mathrm{~A} u\|>0$

(ii) $\mathrm{A} u \neq \mu u, \forall u \in \partial \Omega_{r}, \mu \in[0,1]$.

We first prove that (i) holds. In fact, for any $u \in \partial \Omega_{r}$, we have $0 \leq u(t) \leq r$. By $\left(H_{3}\right)$, the function $f(t, x)$ is nonincreasing relative to $x$ on $[0, r]$ for any $t \in I$, and so

$$
f(t, u(t)) \geq f(t, r) \geq\left(\mu+\varepsilon_{0}\right) r^{p-1}, \quad t \in[0,1]
$$

from (3.12). 
Thus, in view of Lemma 2.4 combined with (3.1) and (3.13), we have

$$
\begin{aligned}
\mathrm{A} u(t) & \geq \frac{1}{(\Gamma(\beta))^{q-1}} \int_{0}^{1} G(t, s)\left(\int_{0}^{s}\left(\mu,+\varepsilon_{0}\right) r^{p-1}(s-\tau)^{\beta-1} d \tau\right)^{q-1} d s \\
& =\frac{\left(\mu+\varepsilon_{0}\right)^{q-1} r}{(\Gamma(\beta+1))^{q-1}} \int_{0}^{1} G(t, s) s^{\beta(q-1)} d s \\
& \geq \frac{\left(\mu+\varepsilon_{0}\right)^{q-1} r}{(\Gamma(\beta+1))^{q-1}} \int_{0}^{1} \eta(s) G(s, s) s^{\beta(q-1)} d s \\
& =\frac{\left(\mu+\varepsilon_{0}\right)^{q-1} Q}{(\Gamma(\beta+1))^{q-1}} r, \quad \text { for all } t \in\left[\eta_{0}, 1\right]
\end{aligned}
$$

where $Q$ is as (2.21). Consequently,

$$
\|\mathrm{A} u\| \geq \frac{\left(\mu+\varepsilon_{0}\right)^{q-1} \mathrm{Q}}{(\Gamma(\beta+1))^{q-1}} r \doteq c_{0}>0 .
$$

Thus $\inf _{u \in \partial \Omega_{r}}\|\mathrm{~A} u\| \geq c_{0}>0$.

(ii) Suppose on the contrary that there exists a $u_{0} \in \partial \Omega_{r}$ and $\mu_{0} \in[0,1]$ such that

$$
\mu_{0} u_{0}=\mathrm{A} u_{0} .
$$

Then, by similar arguments to (3.14), we have

$$
\left\|\mathrm{A} u_{0}\right\| \geq B r,
$$

where $B=\frac{\left(\mu+\varepsilon_{0}\right)^{q-1} Q}{(\Gamma(\beta+1))^{q-1}}$.

By (3.15)-(3.16), we obtain

$$
r=\left\|u_{0}\right\| \geq \mu_{0}\left\|u_{0}\right\|=\left\|A u_{0}\right\| \geq B r .
$$

The hypothesis $\mu=\frac{\Gamma(\beta+1)}{Q^{\frac{1}{q-1}}}$ implies that $B>1$, and so $r>r$ from above inequality, which is a contradiction. That means that (ii) holds.

Hence, applying fixed point index theorem, we have

$$
i\left(A, \Omega_{R}, P\right)=0 .
$$

By (3.11) and (3.17), we have

$$
i\left(A, \Omega_{R} \backslash \Omega_{r}, P\right)=1,
$$

and so, there exists $u_{*} \in \Omega_{R} \backslash \bar{\Omega}_{r}$ with $\mathrm{A} u_{*}=u_{*},\left\|u_{*}\right\|>r$. Hence, $u_{*}$ is a nonnegative solution of BVP (1.5) satisfying $\left\|u_{*}\right\|>r$. Now, we show that $u_{*}(t)>0, t \in(0,1)$.

In fact, since $\left\|u_{*}\right\|>r, u_{*} \in P, G(t, s)>0, t, s \in(0,1)$, from (3.1), we have

$$
\int_{0}^{s} f\left(\tau, u_{*}(\tau)\right)(s-\tau)^{\beta-1} d \tau \not \equiv 0, \quad s \in(0,1)
$$


and so

$$
u_{*}(t)=A u_{*}(t)>0, \quad t \in(0,1)
$$

from the fact that $G(t, s)>0$ and $\int_{0}^{s} f(\tau, u(\tau))(s-\tau)^{\beta-1} d \tau \geq 0, s \in[0,1]$. That is, $u_{*}$ is a positive solution of BVP (1.5).

The proof is complete.

Now, we state another theorem in this article. First, let me introduce some notations which will be used in the sequel.

$$
\text { Let } M_{1}=\frac{\Gamma(\beta+1)}{D^{\frac{1}{q-1}}}, M_{2}=\frac{\Gamma(\beta+1)}{\frac{1}{B_{2}^{q-1}}} \text {, where } D \text { is as }(2.20) \text {. }
$$

Let

$$
B_{2} \frac{\gamma \delta^{2} \sigma\left(1-\eta_{0}\right)^{\alpha+\beta(q-1)+1}}{\Gamma(\alpha-\gamma)}\left[B(\alpha, \beta(q-1)+2)+\left(1-\eta_{0}\right)^{-\gamma} \frac{\sigma \Gamma(\alpha)}{\Gamma(\alpha-\gamma)} B(\alpha-\gamma, \beta(q-1)+2)\right] .
$$

Set $P_{r}=\{u \in P:\|u\|<r\}$, for $r>0$. Let $\omega(u)=\min _{t \in\left[\eta_{0}, 1\right]} u(t)$, for $u \in P$. Obviously, $\omega$ is a nonnegative continuous concave functional on $P$.

Theorem 3.2. Let $\left(H_{1}\right)$ hold. Assume that there exist constants $a, b, c, l_{1}, l_{2}$ with 0 $<a<b<c$ and $l_{1} \in\left(0, M_{1}\right), l_{2} \in\left(M_{2}, \infty\right)$ such that

$\left(D_{1}\right) f(t, x) \leq l_{1} c^{p-1}, x \in[0, c], t \in I ; f(t, x) \leq l_{1} a^{p-1}, x \in[0, a], t \in I$,

$\left(D_{2}\right) f(t, x) \geq l_{1} b^{p-1}, x \in[b, c], t \in\left[\eta_{0}, 1\right]$.

Then BVP (1.5) has at least one nonnegative solution $u_{1}$ and two positive solutions $u_{2}, u_{3}$ with

$$
\left\|u_{1}\right\|<a, b<\min _{t \in\left[\eta_{0}, 1\right]} u_{2}(t), a<\left\|u_{3}\right\|, \min _{t \in\left[\eta_{0}, 1\right]} u_{3}(t)<b .
$$

Proof. By Lemmas 2.3 and 2.4, for $u \in \bar{P}_{\mathcal{C}}$, from (3.1) and condition $\left(D_{1}\right)$, it follows that

$$
\begin{aligned}
\mathrm{A} u(t) & \leq \frac{\left(l_{1}\right)^{q-1} c}{(\Gamma(\beta+1))^{q-1}} \int_{0}^{1} G(s, s) s^{\beta(q-1)} d s \\
& =\frac{\left(l_{1}\right)^{q-1} c}{(\Gamma(\beta+1))^{q-1}} D, \quad t \in I
\end{aligned}
$$

and so

$$
\|\mathrm{A} u\| \leq \frac{\left(l_{1}\right)^{q-1} D}{(\Gamma(\beta+1))^{q-1}} c<c
$$

from the hypothesis $l_{1}<M_{1}$.

Thus, we obtain $A: \bar{P}_{c} \rightarrow P_{c}$. Similarly, we can also obtain $A: \bar{P}_{a} \rightarrow P_{a}$ by condition $\left(D_{1}\right)$. Take $u_{0}=\frac{b+c}{2}$. Then $\omega\left(u_{0}\right)>b$, and so $\{u \in P(\omega, b, c) \mid \omega(u)>b\} \neq \emptyset$.

For any $u \in P(\omega, b, c)$, we have that $u(t) \geq b, t \in\left[\eta_{0}, 1\right]$ and $\|u\| \leq c$. Consequently, by Lemma 2.3, 2.4 and the formula (3.1), for any $t \in\left[\eta_{0}, 1\right]$, it follows from condition $\left(D_{2}\right)$ that 


$$
\begin{aligned}
\mathrm{A} u(t) & =\frac{1}{(\Gamma(\beta))^{q-1}} \int_{0}^{1} G(t, s)\left(\int_{0}^{s} f(\tau, u(\tau))(s-\tau)^{\beta-1} d \tau\right)^{q-1} d s \\
& \geq \frac{1}{(\Gamma(\beta))^{q-1}} \int_{0}^{1} \eta(s) G(s, s)\left(\int_{0}^{s} f(\tau, u(\tau))(s-\tau)^{\beta-1} d \tau\right)^{q-1} d s \\
& \geq \frac{1}{(\Gamma(\beta))^{q-1}} \int_{\eta_{0}}^{1} \eta(s) G(s, s)\left(\int_{0}^{s} f(\tau, u(\tau))(s-\tau)^{\beta-1} d \tau\right)^{q-1} d s \\
& \geq \frac{1}{(\Gamma(\beta))^{q-1}} \int_{\eta_{0}}^{1} \eta(s) G(s, s)\left(\int_{\eta_{0}}^{s} f(\tau, u(\tau))(s-\tau)^{\beta-1} d \tau\right)^{q-1} d s \\
& \geq \frac{l_{2}^{q-1} b}{(\Gamma(\beta+1))^{q-1}} \int_{\eta_{0}}^{1} \eta(s) G(s, s)\left(s-\eta_{0}\right)^{\beta(q-1)} d s .
\end{aligned}
$$

Also, by changing the variable $\theta=\frac{s-\eta_{0}}{1-\eta_{0}}$, we have

$$
\begin{aligned}
& \int_{\eta_{0}}^{1} \eta(s) G(s, s)\left(s-\eta_{0}\right)^{\beta(q-1)} d s \\
= & \frac{\gamma \delta^{2} \sigma}{\Gamma(\alpha-\gamma)} \int_{\eta_{0}}^{1} s\left[(1-s)^{\alpha-1}+\frac{\sigma \Gamma(\alpha)}{\Gamma(\alpha-\gamma)}(1-s)^{\alpha-\gamma-1}\right]\left(s-\eta_{0}\right)^{\beta(q-1)} d s \\
> & \frac{\gamma \delta^{2} \sigma\left(1-\eta_{0}\right)^{\alpha+\beta(q-1)+1}}{\Gamma(\alpha-\gamma)} \int_{0}^{1} \theta^{1+\beta(q-1)}(1-\theta)^{\alpha-1}\left[1+\frac{\sigma \Gamma(\alpha)}{\Gamma(\alpha-\gamma)}\left(1-\eta_{0}\right)^{-\gamma}(1-\theta)^{-\gamma}\right] d \theta \\
= & B_{2},
\end{aligned}
$$

where $B_{2}$ is given by (3.19).

Substituting (3.21) into (3.20), we obtain

$$
\mathrm{A} u(t) \geq \frac{l_{2}^{q-1} B_{2}}{(\Gamma(\beta+1))^{q-1}} b, \quad t \in\left[\eta_{0}, 1\right],
$$

and so $\omega(\mathrm{A} u)>b$ from the hypothesis $l_{2}>M_{2}$.

Summing up the above analysis, we know that all the conditions of Lemma 2.9 with $c=d$ are satisfied, and so BVP (1.5) has at least three nonnegative solutions $u_{1}, u_{2}, u_{3}$ with

$$
\left\|u_{1}\right\|<a, b<\min _{t \in\left[\eta_{0}, 1\right]} u_{2}(t), a<\left\|u_{3}\right\|, \min _{t \in\left[\eta_{0}, 1\right]} u_{3}(t)<b .
$$

By similar argument to (3.18), we can deduce that $u_{2}$ and $u_{3}$ are two positive solutions.

The proof is complete.

Example 3.1. Consider the following BVP

$$
\left\{\begin{array}{l}
D_{0+}^{\beta}\left(\varphi_{p}\left(D_{0+}^{\alpha} u\right)\right)(t)+\left(t^{2}+\cos ^{2} u\right)=0, \quad 0<t<1, \\
u(0)=0, \quad u(1)+\sigma D_{0+}^{\gamma} u(1)=0, \quad D_{0+}^{\alpha} u(0)=0,
\end{array}\right.
$$


where $1<\alpha<2,0<\beta<1,0<\gamma<1,0 \leq \alpha-\gamma-1, \sigma>0$ and the $p$-Laplacian operator is defined as $\phi_{p}(s)=|s|^{p-2} s, p>1$.

It is easy to verify that all assumptions of Theorem 3.1 are satisfied. Hence, by the conclusion of Theorem 3.1, BVP (3.22) has at least one positive solution on [0, 1].

Example 3.2. Consider the following BVP

$$
\begin{cases}D_{0+}^{\frac{1}{2}}\left(\varphi_{\frac{3}{2}}\left(D_{0+}^{\frac{3}{2}} u\right)\right)(t)+f(t, u)=0, & 0<t<1, \\ u(0)=0, \quad u(1)+\frac{2}{\sqrt{\pi}} D_{0+}^{\frac{1}{2}} u(1)=0, \quad D_{0+}^{\frac{3}{2}} u(0)=0\end{cases}
$$

where $\alpha=\frac{2}{3}, \beta=\gamma=\frac{1}{2}, \sigma=\frac{2}{\sqrt{\pi}}, p=\frac{3}{2}$ relative to Theorem 3.2. With the aid of computation we have that $M_{1}=\sqrt{\pi} \sqrt[4]{\pi} \sqrt{\frac{20}{32+5 \pi}}=1.527 \ldots, M_{2}=\frac{64}{15} \sqrt{\pi} \sqrt[4]{\pi}\left(\frac{7}{35+4 \sqrt{15}}\right)^{\frac{1}{2}}=3.74 \ldots, \eta_{0}=\frac{1}{16}$. Take $l_{1}=$ $1.5, l_{2}=4$. Then $l_{1} \in\left(0, M_{1}\right), l_{2} \in\left(M_{2}, \infty\right)$. Again choosing $a=\frac{\sqrt[3]{2.25}}{4}, b=1, c=196$, and setting

$$
f(t, x)=\left\{\begin{array}{l}
\left(\sin \frac{\pi}{90}(32 t+13)\right)\left(x^{\frac{1}{2}}+7\right), \quad x \in[1,+\infty), \\
8\left(\sin \frac{\pi}{90}(32 t+13)\right) x^{2}, \quad x \in[0,1),
\end{array}\right.
$$

for $t \in[0,1]$, then we see that $f$ satisfies the following relations:

$$
\begin{aligned}
& f(t, x) \leq 21=1.5 \times 196^{\frac{1}{2}}=l_{1} c^{p-1}, \quad t \in[0,1], \quad x \in[0,196]=[0, c] \\
& f(t, x) \geq 4=l_{2} b^{p-1}, \quad t \in\left[\frac{1}{16}, 1\right]=\left[\eta_{0}, 1\right], \quad x \in[1,196]=[b, c] \\
& f(t, x) \leq \frac{3 \sqrt[3]{1.5}}{4}=1.5 \times \frac{\sqrt[3]{1.5}}{2}=l_{1} a^{p-1}, \quad t \in[0,1], \quad t \in\left[0, \frac{\sqrt[3]{2.25}}{4}\right]=[0, a] .
\end{aligned}
$$

So, all the assumptions of Theorem 3.2 are satisfied. By Theorem 3.2, we arrive at BVP (3.23) has at least one nonnegative solution $u_{1}$ and two positive solutions $u_{2}, u_{3}$ with

$$
\left\|u_{1}\right\|<\frac{\sqrt[3]{2.25}}{4}, 1<\min _{t \in\left[\eta_{0}, 1\right]} u_{2}(t), \frac{\sqrt[3]{2.25}}{4}<\left\|u_{3}\right\|, \min _{t \in\left[\eta_{0}, 1\right]} u_{3}(t)<1
$$

\section{Acknowledgements}

The author sincerely thanks the anonymous referees for their valuable suggestions and comments which have greatly helped improve this article. Supported by the Natural Science Foundation of Educational Committee of Hubei Province (D200722002).

\section{Competing interests}

The author declares that they have no competing interests.

Received: 12 October 2011 Accepted: 15 February 2012 Published: 15 February 2012

\section{References}

1. Glockle, WG, Nonnenmacher, TF: A fractional calculus approach of self-similar protein dynamics. Biophys J. 68, 46-53 (1995). doi:10.1016/S0006-3495(95)80157-8

2. Hilfer, R: Applications of Fractional Calculus in Physics. World Scientific, Singapore (2000) 
3. Metzler, F, Schick, W, Kilian, HG, Nonnenmacher, TF: Relaxation in filled polymers: a fractional calculus approach. J Chem Phys. 103, 7180-7186 (1995). doi:10.1063/1.470346

4. Podlubny, I: Fractional Differential Equations. Academic Press, San Diego (1999)

5. Podlubny, l: Geometric and physical interpretation of fractional integration and fractional differentiation. Fract Calc Appl Anal. 5, 367-386 (2002)

6. Kilbas, AA, Srivastava, HM, Trujillo, Jj: Theory and Applications of Fractional Differential Equations. In North-Holland Mathematics Studies, vol. 204,Elsevier, Amsterdam (2006)

7. Lakshmikantham, V, Leela, S, Vasundhara, J: Theory of Fractional Dynamic Systems. Cambridge Academic Publishers, Cambridge (2009)

8. Samko, SG, Kilbas, AA, Marichev, Ol: Fractional Integrals and Derivaes Theory and Applications. Gordon and Breach Science Publisher, Yverdon (1993)

9. Agarwal, RP, Benchohra, M, Hamani, S: A survey on existence result for boundary value problems of nonlinear fractional differential equations and inclusions. Acta Appl Math. 109, 973-1033 (2010). doi:10.1007/s10440-008-9356-6

10. Agarwal, RP, O'Regan, D, Stanek, S: Positive solutions for Dirichlet problems of singular nonlinear fractional differential equations. J Math Anal Appl. 371, 57-68 (2010). doi:10.1016/j.jmaa.2010.04.034

11. Kosmatov, N: Integral equations and initial value problems for nonlinear differential equations of fractional order. Nonlinear Anal. 70, $2521-2529$ (2009). doi:10.1016/j.na.2008.03.037

12. Christopher, SG: Existence of a positve solution to a class of fractional differential equations. Appl Math Lett. 23 1050-1055 (2010). doi:10.1016/j.aml.2010.04.035

13. Tian, Y, Bai, Z: Existence results for the three-point impulsive boundary value problem involving fractional differential equations. Comput Math Appl. 59, 2601-2609 (2010). doi:10.1016/j.camwa.2010.01.028

14. Deng, J, Ma, L: Existence and uniqueness of solutions of initial value problems for nonlinear fractional differential equations. Appl Math Lett. 23, 676-680 (2010). doi:10.1016/j.aml.2010.02.007

15. Zhao, Y, Sun, S, Han, Z, Zhang, M: Positive solutions for boundary value problems of nonlinear fractional differential equations. Appl Math Comput. 217, 6950-6958 (2011). doi:10.1016/j.amc.2011.01.103

16. Kaufmann, ER, Mboumi, E: Positive solutions of a boundary value problem for a nonlinear fractional differential equation. Electron J Qual Theory Diff Equ. 3, 1-11 (2008)

17. Wang, X: Impulsive boundary value problem for nonlinear differential equations of fractional order. Comput Math Appl. 62, 2383-2391 (2011). doi:10.1016/j.camwa.2011.07.026

18. Zhou, W, Chu, Y: Existence of solutions for fractional differential equations with multipoint boundary conditions. Commun Nonlinear Sci Numer Simulat. 17, 1142-1148 (2012). doi:10.1016/j.cnsns.2011.07.019

19. Chai, G: Existence results for boundary value problems of nonlinear fractional differential equations. Comput Math Appl. 62, 2374-2382 (2011). doi:10.1016/j.camwa.2011.07.025

20. Caballero, J, Harjani, J, Sadarangani, K: On existence and uniqueness of positive solutions to a class of fractional boundary value problems. Bound Value Probl 25 (2011). 2011

21. Ahmad, B, Nieto, Jj: Riemann-Liouville fractional integro-differential equations with fractional nonlocal integral boundary conditions. Bound Value Probl 36 (2011). 2011

22. Yang, W: Positive solutions for a coupled system of nonlinear fractional differential equations with integral boundary conditions. Comput Math Appl. 63, 288-297 (2012). doi:10.1016/j.camwa.2011.11.021

23. Agarwa, RP, Ahmad, B: Existence theory for anti-periodic boundary value problems of fractional differential equations and inclusions. Comput Math Appl. 62, 1200-1214 (2011). doi:10.1016/j.camwa.2011.03.001

24. Wang, J, Xiang, H, Liu, Z: Positive solutions for three-point boundary value problems of nonlinear fractional differential equations with p-Laplacian. Far East J Appl Math. 37, 33-47 (2009)

25. Wang, J, Xiang, H, Liu, Z: Upper and lower solutions method for a class of singular fractional boundary value problems with p-Laplacian operator. Abst Appl Anal 12 (2010). 2010, (Article ID 971824)

26. Feng, $M, Z$ Zhang, $X, G e, W$ : New existence results for higher-order nonlinear fractional differential equation with integral boundary conditions. Bound Value Probl 20 (2011). 2011 (Article ID 720702)

27. Mena, JC, Harjani, J, Sadarangani, K: Existence and uniqueness of positive and nonde-creasing solutions for a class of singular fractional boundary value problems. Bound Value Probl 10 (2009). 2009 (Article ID 421310)

28. Arara, A, Benchohra, M, Hamidi, N, Nieto, Jj: Fractional order differential equations on an unbounded domain. Nonlinear Anal. 72, 580-586 (2010). doi:10.1016/j.na.2009.06.106

29. Yang, C, Yan, J: Positive solutions for third-order Sturm-Liouville boundary value problems with $p$-Laplacian. Comput Math Appl. 59, 2059-2066 (2010). doi:10.1016/j.camwa.2009.12.011

30. Anderson, DR, Avery, Rl, Henderson, J: Existence of solutions for a one-dimensional p-Laplacian on time scales. J Diff Equ Appl. 10, 889-896 (2004). doi:10.1080/10236190410001731416

31. Goodrich, CS: The existence of a positive solution to a second-order delta-nabla $p$-Laplacian BVP on a time scale. Appl Math Lett. 25, 157-162 (2012). doi:10.1016/j.aml.2011.08.005

32. Graef, JR, Kong, L: First-order singular boundary value problems with $p$-Laplacian on time scales. J Diff Equ Appl. 17, 831-839 (2011). doi:10.1080/10236190903443111

33. Goodrich, CS: Existence of a positive solution to a first-order $p$-Laplacian BVP on a time scale. Nonlinear Anal. 74 1926-1936 (2011). doi:10.1016/j.na.2010.10.062

34. Bai, Z, Lü, H: Positive solutions for boundary value problem of nonlinear fractional differential equation. J Math Anal Appl. 311, 495-505 (2005). doi:10.1016/j.jmaa.2005.02.052

35. Leggett, RW, Williams, LR: Multiple positive solutions of nonlinear operators on ordered Banach spaces. Indiana Univ Math J. 28, 673-688 (1979). doi:10.1512/iumj.1979.28.28046

doi:10.1186/1687-2770-2012-18

Cite this article as: Chai: Positive solutions for boundary value problem of fractional differential equation with $p$ Laplacian operator. Boundary Value Problems 2012 2012:18. 